\title{
Identification of circulating microRNAs as diagnostic biomarkers for use in multiple myeloma
}

\author{
CI Jones', MV Zabolotskaya', AJ King', HJS Stewart', GA Horne', TJ Chevassut' and SF Newbury*,I \\ 'Medical Research Building, Brighton and Sussex Medical School, University of Sussex, Falmer, Brighton BNI 9PS, UK
}

\begin{abstract}
BACKGROUND: Multiple myeloma is a plasma cell disorder that is characterised by clonal proliferation of malignant plasma cells in the bone marrow, monoclonal paraprotein in the blood or urine and associated organ dysfunction. It accounts for approximately $1 \%$ of cancers and I $3 \%$ of haematological cancers. Myeloma arises from an asymptomatic proliferation of monoclonal plasma cells termed monoclonal gammopathy of undetermined significance (MGUS).

METHODS: MicroRNA expression profiling of serum samples was performed on three patient groups as well as normal controls. Validation of the nine microRNAs detected as promising biomarkers was carried out using TaqMan quantitative reverse transcription PCR. MicroRNA levels in serum were normalised using standard curves to determine the numbers of microRNAs per $\mu$ l of serum. RESULTS: Three serum microRNAs, miR-720, miR-I308 and miR-1246, were found to have potential as diagnostic biomarkers in myeloma. Use of miR-720 and miR-I 308 together provides a powerful diagnostic tool for distinguishing normal healthy controls, as well as patients with unrelated illnesses, from pre-cancerous myeloma and myeloma patients. In addition, the combination of miR-I246 and miR-I 308 can distinguish MGUS from myeloma patients.

CONCLUSION: We have developed a biomarker signature using microRNAs extracted from serum, which has potential as a diagnostic and prognostic tool for multiple myeloma.

British Journal of Cancer (2012) I 07, 1987-1996. doi:10.1038/bjc.2012.525 www.bjcancer.com
\end{abstract}

Published online 20 November 2012

(c) 2012 Cancer Research UK

Keywords: myeloma; microRNAs; biomarkers; diagnostics; cleaved tRNA; serum miRNAs

Multiple myeloma is a malignant disorder of bone marrow plasma cells that accounts for approximately $13 \%$ of all haematological cancers and 1\% of all cancers (Terpos and Rahemtulla, 2005; Kyle and Rajkumar, 2008). In multiple myeloma, malignant plasma cells, which normally proliferate at a low rate, undergo a massive clonal expansion resulting in high levels of production of monoclonal immunoglobulin (Ig) (M-protein) or an Ig chain (M-component) (Terpos and Rahemtulla, 2005) in the blood. Because malignant plasma cells progressively infiltrate the bone marrow, their proliferation interferes with cell signalling pathways involved in osteoblast formation. The uncoupling of bone resorption and formation leads to a rapid bone loss, osteoporosis, lytic lesions and fractures. Hypercalcaemia as a result of loss of calcium from the bone can cause life-threatening dehydration and renal failure. Myeloma patients usually also have reduced levels of normal Igs, resulting in severe immunodeficiency and susceptibility to bacterial infections. Although myeloma is presently incurable, recent advances in treatment, including the use of thalidomide and newer agents such as the immunomodulatory drug lenalidomide and the proteosome inhibitor bortezomib, as well as autologous stem cell transplantation, can improve life expectancy (Terpos and Rahemtulla, 2005; Kyle and Rajkumar, 2008).

*Correspondence: Dr SF Newbury; E-mail: s.newbury@bsms.ac.uk Received 13 September 2012; revised 29 October 2012; accepted 30 October 2012; published online 20 November 2012
Myeloma usually progresses from an asymptomatic precancerous stage of clonal plasma cell proliferation termed 'monoclonal gammopathy of undetermined significance' (MGUS). The MGUS is present in more than $3 \%$ of the population above the age of 50 and progresses to myeloma or a related malignancy at a rate of $\sim 1 \%$ per year (McKenna et al, 2008). A variant of MGUS is asymptomatic or smouldering plasma cell myeloma where the diagnostic criteria for multiple myeloma are met but there is no related organ or tissue impairment (Kyle et al, 2007). Multiple myeloma is characterised by various cytogenetic abnormalities, some of which can be of prognostic significance. However, the relevance of these chromosomal abnormalities to disease progression is unclear and none provide reliable predictors of survival (McKenna et al, 2008). Because no single factor can identify those patients with MGUS likely to progress to myeloma, patients need to be monitored at regular intervals. Therefore, a diagnostic method to identify those patients who are most at risk of cancer progression would be of immense benefit to patients and would raise the possibility of early treatment and improved prognosis.

Recently, a number of groups have investigated the efficacy of microRNAs (miRNAs) as diagnostic or prognostic biomarkers. miRNAs are small, non-coding RNAs that are known to regulate gene expression (Jones and Newbury, 2010; Zen and Zhang, 2010; Reid et al, 2011). At present, there are known to be 2042 (miRBase 19.0, Aug 2012) miRNAs encoded in the human genome, many of which are specifically expressed in particular tissues. miRNAs can bind to target mRNAs, usually in their $3^{\prime}$ untranslated regions, and cause downregulation of protein expression by translational 
repression or cleavage and degradation of the target mRNA (Kong et al, 2012). Previous studies, using bone marrow plasma cells, have suggested dysregulation of a set of miRNAs in myeloma, including miR-181a/b, miR-17-92, miR-32, miR-193b-365, miR192-194-215 and miR-15a/16 (Pichiorri et al, 2008; Lionetti et al, 2009; Roccaro et al, 2009). These studies also found dysregulation of miRNAs in MGUS patients (i.e., miR-21, miR-106-25, miR-181a/ b, miR-1 and miR-133). Although these changes in miRNA levels shed light on the molecular mechanisms of multiple myeloma malignancies, they are of limited use for a minimally invasive test as the changes take place within bone marrow plasma cells.

The use of miRNAs as biomarkers has greatly increased as a result of the discovery that they are present in the circulating blood. A number of groups have shown that miRNAs can be detected in human serum or plasma, where they are thought to be protected from degradation by being encapsulated in microvesicles or exosomes and/ or are bound by RNA-binding proteins such as Ago2 and nucleophosmin (Reid et al, 2011; Chen et al, 2012). Changes in levels of particular subsets of circulating miRNAs have been associated with various cancers. For example, in breast cancer patients, miR-195 and let-7a have been found to be significantly upregulated in the sera of patients with breast cancer compared with healthy, age-matched subjects, whereas no significant difference was seen in levels of miR155 and miR-10b (Heneghan et al, 2010). These data therefore show that it is feasible to use miRNAs derived from blood samples as a minimally invasive diagnostic tool for cancer patients.

In the present study, we evaluated miRNA expression patterns in the serum from the circulating blood derived from multiple myeloma patients, MGUS patients and controls. We first used microarray analysis to identify particular miRNAs that were differentially expressed in pools of samples derived from each participant group. We then examined individual samples, using TaqMan real-time quantitative reverse transcription PCR (qRTPCR) to assess the abundance of particular mature miRNAs in each sample. Using these methods we found three miRNAs, which have potential as biomarkers in myeloma. Our data show that circulating miRNAs provide a basis for a convenient and noninvasive diagnostic test, which has potential prognostic value.

\section{MATERIALS AND METHODS}

\section{Patient and control samples}

For the initial identification of biomarkers, we used myeloma patients, pre-cancerous MGUS patients, non-myeloma, non-MGUS hospitalised patients and normal healthy controls without a family history of haematological disease. All myeloma, MGUS and nonmyeloma, non-MGUS hospitalised patients were assessed by serum/urine electrophoresis and serum-free light chain detection, along with routine full blood count and biochemical profile (Kyle and Rajkumar, 2008; McKenna et al, 2008). Patients with myeloma and MGUS were subject to a bone marrow biopsy and skeletal survey to stage their condition as per standard practice. The nonmyeloma, non-MGUS patients, who were admitted to the Brighton and Sussex University Hospital because of similar symptoms to that of myeloma patients, had no detectable paraprotein in their serum. Relevant details on the patients used are given in Table 1. Patient/control blood samples were collected in VACUETTE Gel Z Serum Sep Clot Activator (Greiner Bio-One, Kremsmüenster, Austria) $5 \mathrm{ml}$, red/yellow tubes, and the serum was removed according to normal hospital procedures. Serum samples were stored at $4{ }^{\circ} \mathrm{C}$ for less than 7 days before freezing at $-80^{\circ} \mathrm{C}$. All samples from human participants were collected with informed written consent, and the research was carried out under the 'Brighton blood disorder study', which has ethical and $\mathrm{R}+\mathrm{D}$ approval for donation of blood and marrow samples for research purposes (references: 09/025/CHE and 09/H1107/1).
Table I Summary of clinical details of human participants used for microarray analysis of serum samples

\begin{tabular}{lc}
\hline Patient groups & $\mathbf{n}(\%)$ \\
\hline Normal healthy controls (N) & 13 \\
Total & $5(38.5)$ \\
$\quad$ Male & $8(61.5)$ \\
Female & $42-58$ \\
Age on collection of sample & $47.7 \pm 5.7$ \\
Range & \\
Mean and s.d. & 20 \\
Normal hospitalised (NH) controls & $9(45)$ \\
Total & $11(55)$ \\
Male & $41-93$ \\
Female & $76.1 \pm 13.2$ \\
Age on collection of sample & \\
Range & 15 \\
Mean and s.d. & $11(73)$ \\
MGUS (MG) patients & $4(27)$ \\
Total & \\
Male & $50-81$ \\
Female & $64.5 \pm 11.2$ \\
Age on collection of sample & \\
Range & \\
Mean and s.d. & 24 \\
Myeloma (M) patients & $12(50)$ \\
Total & $12(50)$ \\
Male & Female \\
Age on collection of sample & $58-89$ \\
Range & $73.5 \pm 7.8$ \\
Mean and s.d. & \\
\hline &
\end{tabular}

\section{RNA extraction}

Total RNA extraction was performed using a mirVana PARIS kit (Life Technologies, Carlsbad, CA, USA), following the manufacturer's instructions. Samples were not enriched for miRNAs. The yields of total RNA were $140-360$ ng per $400 \mu \mathrm{l}$ of serum.

\section{Microarrays}

Agilent Human miRNA $8 \times 15 \mathrm{~K}$ Microarrays (Agilent, Santa Clara, CA, USA, V3, miRBase version 12.0) were used according to the manufacturer's instructions. Hybridisation was performed using Agilent hybridisation chambers in a rotary UVP HB-1000 oven. The slides were scanned using an Axon GenePix 4000B Microarray Scanner and analysed using GenePix Pro 6.0 (Molecular Devices, Sunnyvale, CA, USA). Two arrays were used for each pooled sample; because there were four pooled samples, this experiment used one microarray slide of eight arrays. Each array contained 16 spots per miRNA, therefore 32 spots in total for each miRNA were analysed for the four sample groups. On each array, a miRNA was taken to have been detected if more than four of its spots were visible above the background level. This equates to at least 8 out of 32 spots over both arrays. Microarray data has been deposited in the ArrayExpress Archive, accession number E-MTAB-1254.

\section{Quantitative PCR}

TaqMan miRNA assays (Life Technologies) were used for qRT-PCR. Reverse transcription was performed using a TaqMan MicroRNA Reverse Transcription Kit (Life Technologies), and the specific looped RT primers provided with was TaqMan assay. The qRT-PCR was performed using the specific primer/probe combination provided with each TaqMan assay and TaqMan Universal PCR Master Mix, No AmpErase UNG (Life Technologies). For 
absolute quantification, synthetic miRNAs from Sigma-Aldrich (St Louis, MO, USA) were used for standards. Two RT reactions and two qPCRs were performed per RNA preparation from serum (four technical replicates total). One RT reaction and three qPCRs were performed for each standard (three technical replicates total).

\section{RESULTS}

\section{Optimisation of RNA extraction techniques}

Our search for a myeloma biomarker first required us to determine the most robust and reliable method for extraction of miRNAs from human serum. Serum, rather than plasma was the starting material of choice because serum is normally used in the clinic in serum electrophoresis tests to assess levels of paraprotein. miRNAs are usually found at low levels in serum, therefore optimisation of extraction was important. We tried three extraction methods: Trizol/chloroform followed by isopropanol precipitation (using $500 \mu \mathrm{l}$ of serum), the mirVana PARIS kit (Life Technologies) (using $400 \mu \mathrm{l}$ of serum) and the Exiqon's protocol using miRNeasy mini kit (Qiagen, Hilden, Germany) (using $250 \mu \mathrm{l}$ of serum). Nonmyeloma, non-MGUS patients were chosen for this initial study. Total RNA was extracted in triplicate, the yield and quality were assessed using a Nanodrop spectrophotometer and the presence of a particular mature miRNA (miR-181b) was assessed using TaqMan real-time qRT-PCR. Of the three extraction methods used, the mirVANA PARIS kit proved to provide the most consistent results, with the highest yield of miRNA, as assessed by the levels of miR-181b.

\section{Identification of potential miRNA biomarkers using microarrays}

The next step was to identify miRNAs in serum from myeloma and MGUS patients, which could act as a robust and reliable biomarker for the disease. For this step, we chose to use miRNA microarrays, as this platform identifies miRNAs by hybridisation to immobilised nucleic acids, rather than using a PCR step. As each method can have inherent bias (Git et al, 2010), we chose to initially identify miRNAs using microarrays and then follow-up using a PCR-based method.

To optimise this detection step, and to identify the miRNAs most likely to be dysregulated, we chose to pool samples within patient and control groups. This pooling method had the advantage that we would only detect miRNAs that were expressed in the majority of patients/subjects within the group. Therefore, this procedure reduced variation between individuals and enriched for miRNAs most likely to change between patient and control groups. The individuals used per group were not more than 20. The four groups of serum samples comprised normal, healthy controls $(n=13)$, non-MGUS/nonmyeloma patients $(n=20)$, patients who had low levels of paraprotein in their serum (IgG, IgA $\left.<10 \operatorname{gl}^{-1}(n=20)\right)$ and patients who had high paraprotein in their serum (IgG, IgA $\left.>20 \mathrm{gl}^{-1}(n=19)\right)$. These levels of paraprotein were used as a criterion to clearly distinguish between patient groups. The use of paraprotein levels to separate the latter two groups provided an easy, quantitative way of distinguishing these two groups of patients. RNA was extracted in duplicate from the pooled serum samples using the mirVana PARIS kit as described above.

After the relevant quality control steps (see Materials and Methods), nine miRNAs were reliably detected in all patient/ control groups by the Agilent Human miRNA arrays. These miRNAs, listed in Table 2, were detected in at least two of the patient or control groups. Note that our pooling method meant that miRNAs expressed at low levels or expressed in only a few individuals would not be detected. This enrichment process therefore allowed us to concentrate on the few miRNAs that were
Table 2 miRNAs detected in pooled patient and control samples, as detected by Agilent Human miRNA microarrays

\begin{tabular}{ll}
\hline miRNA & Sequence \\
\hline hsa-miR-45I & 5'-AAACCGUUACCAUUACUGAGUU-3' \\
hsa-miR-638 & 5'-AGGGAUCGCGGGCGGGUGGCGGCCU-3' \\
hsa-miR-720 & 5'-UCUCGCUGGGGCCUCCA-3' \\
hsa-miR-I246 & 5'-AAUGGAUUUUUGGAGCAGG-3' \\
hsa-miR-1308 & 5'-GCAUGGGUGGUUCAGUGG-3' \\
hsa-miR-1915 & 5'-CCCCAGGGCGACGCGGCGGG-3' \\
hsa-miR-1974 & 5'-UGGUUGUAGUCCGUGCGAGAAUA-3' \\
hsa-miR-574-5p & 5'-UGAGUGUGUGUGUGUGAGUGUGU-3' \\
hsa-miR-762 & 5'-GGGGCUGGGGCCGGGGCCGAGC-3' \\
\hline
\end{tabular}

All of these showed potential as biomarkers as the levels of miRNAs varied between the patient and control groups. The expression levels of six of these miRNAs (in bold) were verified using TaqMan qRT-PCR. Three miRNAs (miR-720, miR-I246 and miR-1308) chosen for further analysis in individual patient samples. TaqMan qRT-PCR assays for the remaining three miRNAs were not available or were unreliable.

most likely to change between groups, as well as be detected at reasonable levels in all individual samples. Our method of selecting miRNAs for further study was also very rigorous; miRNAs were only selected if the signal on the array was significantly above background levels in at least 4 out of 16 spots (per miRNA per array). The levels of these miRNAs were not compared between groups using the array data as there is no known internal control in serum for use as a normaliser to compensate for technical variations between arrays.

\section{Validation of array results using real-time RT-PCR}

To validate the microarray results and confirm the pattern of miRNA expression between patient/control samples, TaqMan qRT-PCR assays were employed. The qRT-PCR is more sensitive and more quantitative over a greater dynamic range than microarrays. TaqMan assays were available and worked reliably for six of the nine miRNAs detected on the arrays. These six assays were tested on each of the replicated RNA samples (i.e., N1/N2 etc.) from pooled serum used on the arrays. Two reversetranscriptase replicates and two PCR replicates were carried out for each pooled sample, giving eight replicates for each pool in total. All six miRNAs were detected in both replicates of each group with the expression pattern of each miRNA being different for each patient group. Of these six potential miRNA biomarkers, three were chosen for further investigation; miR-720, miR-1246 and miR-1308. These were selected because they are expressed at reasonably high levels (i.e., at Cts of 19-27) and show different patterns of expression between patient and control groups. Note that recent re-evaluation of the miRNAs in the miRbase database has shown that miR-1308 is not a miRNA but is a $5^{\prime}$-cleaved fragment of a GlyGCC tRNA. Although accurate quantification of expression differences between samples is not possible because of the lack of a normaliser miRNA, it seems likely that these differences reflect true biological differences as the technical variation between $\mathrm{qPCR}$ tests was minimal.

\section{Absolute quantitation of miRNA levels in individual serum samples}

At present, there is no reliable normaliser (or reference control) available for use in qRT-PCR assays on miRNA samples extracted from human serum. The mRNAs that are often used for analysis of gene expression in tissues (e.g., GAPDH) are not found in circulating blood, presumably because they are not protected by microvesicles/exosomes. Small non-coding nuclear RNAs, such as 
RNU44, which are often used as normaliser RNAs in qRT-PCR experiments, have been shown by ourselves and others to be absent from serum samples (Zhu et al, 2009). Although some snoRNAs, snRNAs and rRNAs can be detected in serum, their expression is quite variable (Hunter et al, 2008). miR-16 has been used as a normaliser in some studies, but we did not find it to be expressed at similar levels between patient and control samples. Other groups have standardised miRNA levels according to the total amount of RNAs extracted from a particular quantity of serum, but this is not satisfactory, as patients with cancer can have significantly more total miRNAs in their serum than normal controls (Lodes et al, 2009). Spike-in controls, often using Caenorhabditis elegans miRNAs, which are not found in humans (e.g., cel-miR-39) (Mitchell et al, 2008) can be used to determine the miRNA extraction efficiency. However, these are of limited value when comparing miRNA levels between patients.

To accurately determine the expression differences between groups, we performed absolute quantification to determine the copy number of each miRNA per $\mu$ l of serum. At present, this appears to be the only way of accurately determining the differences in miRNA levels between individual patients and controls. Absolute quantification requires standard samples to be run alongside unknown samples for comparison. The standards used must be the miRNA of interest at a known concentration, for example, miRNAs produced synthetically. The synthetic miRNA is diluted across a range of several orders of magnitude, and these dilutions are treated in the same way as the unknown samples. The standard samples are used to plot a curve of cycle threshold (Ct) vs copy number, and the $\mathrm{Ct}$ of the unknown samples is compared with this to give a copy number. The copy numbers from individuals can then be compared between groups to show differences in expression.

\section{Comparison of levels of miR-720, miR-1245 and miR-1308 in individual patients}

In order to determine the pattern of miRNA expression in serum in individual patients and controls, RNA was prepared from $200 \mu \mathrm{l}$ of serum from the individual patients/controls that had formed the pools for earlier experiments. Two RT reactions were performed per patient/control followed by two technical replicates for each (four technical replicates per individual/miRNA combination). The absolute amounts of each miRNA, per $\mu \mathrm{l}$ of serum in each patient sample were determined as above using the corresponding synthetic miRNA to generate the standard curve (Figure 1).

As can be seen from Figure 1, the pattern of expression of each miRNA differs between patient groups. For miR-720, the levels are significantly higher in myeloma and MGUS patients compared with normal controls, whereas the levels of miR-1308 are significantly lower in patients compared with normal controls. The different patterns of expression of miRNAs suggest independent control of each miRNA by the cells secreting the miRNAs. Second, for all three miRNAs, the levels of miRNAs are much more tightly grouped in the normal controls compared with the patient groups. These data suggest that levels of these miRNAs in serum are normally tightly controlled and are dysregulated in disease. Our results further suggest that miRNAs can be used as a diagnosis test for MGUS and myeloma.

The non-MGUS, non-myeloma group show a wider range of expression compared with the other groups. These patients had no detectable paraprotein in their blood, and were subsequently diagnosed with a variety of illnesses unrelated to myeloma. These illnesses included hypercalcaemia attributable to underlying malignancy and patients with anaemia associated with renal failure. Various malignancies and renal impairment, in particular chronic renal impairment, have previously been shown to be associated with distinct miRNA signature in serum (Neal et al, 2011). Therefore, the range of miRNA expression in these patients is likely to reflect the wide range of diseases from which they are suffering.

The graphs also show that the pattern of expression of each of the three miRNAs, miR-720, miR-1246 and miR-1308, are similar between MGUS and myeloma patients. This is to be expected as MGUS is well established as a pre-cancerous state for myeloma. It is also interesting to note that the miRNAs we have detected as biomarkers in the serum are different from those dysregulated in plasma cells (Pichiorri et al, 2008; Lionetti et al, 2009; Roccaro et al, 2009).
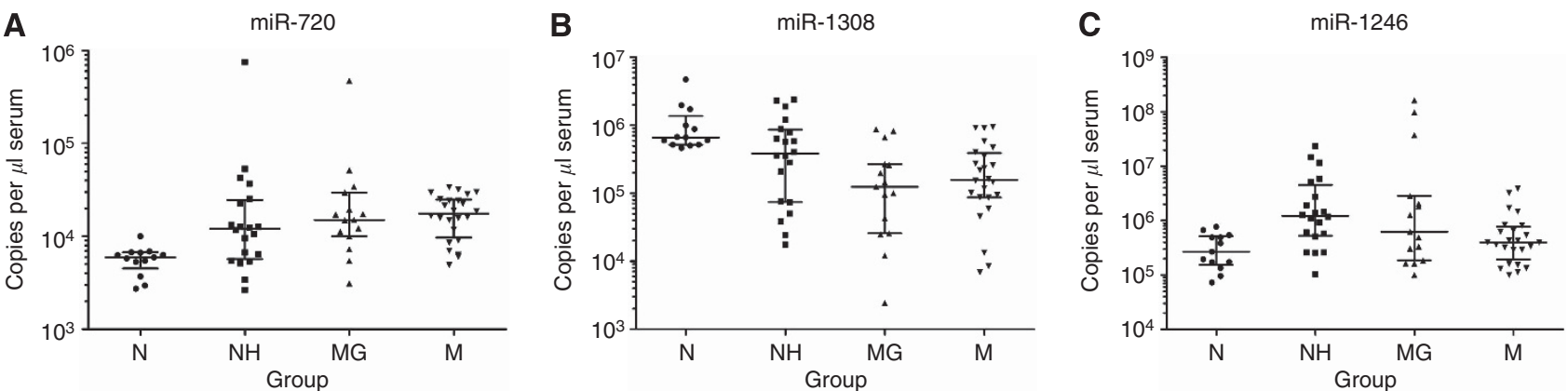

\begin{tabular}{|l|l|}
\hline Comparison & $P$-value \\
\hline $\mathrm{N}$ vs $\mathrm{NH}$ & $<0.05^{*}$ \\
\hline $\mathrm{N}$ vs $\mathrm{MG}$ & $<0.01^{* *}$ \\
\hline $\mathrm{N}$ vs $\mathrm{M}$ & $<0.001^{* * *}$ \\
\hline $\mathrm{NH}$ vs $\mathrm{MG}$ & $\mathrm{ns}$ \\
\hline $\mathrm{NH}$ vs $\mathrm{M}$ & $\mathrm{ns}$ \\
\hline $\mathrm{MG}$ vs $\mathrm{M}$ & $\mathrm{ns}$ \\
\hline
\end{tabular}

\begin{tabular}{|l|l|}
\hline Comparison & $P$-value \\
\hline $\mathrm{N}$ vs $\mathrm{NH}$ & $\mathrm{ns}$ \\
\hline $\mathrm{N}$ vs $\mathrm{MG}$ & $<0.01^{\text {** }}$ \\
\hline $\mathrm{N}$ vs $\mathrm{M}$ & $<0.01^{\text {** }}$ \\
\hline $\mathrm{NH}$ vs $\mathrm{MG}$ & $\mathrm{ns}$ \\
\hline $\mathrm{NH}$ vs $\mathrm{M}$ & $\mathrm{ns}$ \\
\hline $\mathrm{MG}$ vs $\mathrm{M}$ & $\mathrm{ns}$ \\
\hline
\end{tabular}

\begin{tabular}{|l|l|}
\hline Comparison & $P$-value \\
\hline $\mathrm{N}$ vs $\mathrm{NH}$ & $<0.01^{* *}$ \\
\hline $\mathrm{N}$ vs $\mathrm{MG}$ & $\mathrm{ns}$ \\
\hline $\mathrm{N}$ vs $\mathrm{M}$ & $\mathrm{ns}$ \\
\hline $\mathrm{NH}$ vs $\mathrm{MG}$ & $\mathrm{ns}$ \\
\hline $\mathrm{NH}$ vs $\mathrm{M}$ & $\mathrm{ns}$ \\
\hline $\mathrm{MG}$ vs $\mathrm{M}$ & $\mathrm{ns}$ \\
\hline
\end{tabular}

Figure I Comparison of the serum levels of miR-720 (A), miR-1308 (B) and miR-1246 (C) in Normal (N), Normal hospitalised (NH), MGUS (MG) and myeloma (M) groups. Graphs show median level with interquartile range. The Kruskal-Wallis test with Dunn's post test was used to determine the significance of differences between groups. The serum levels were determined using TaqMan miRNA qRT-PCR following RNA extraction. Two technical replicates were performed on two cDNA replicates (four technical replicates total per sample/miRNA combination. 
miR-720 and miR-1308 provide a biomarker signature, which can distinguish MGUS and myeloma patients from normal healthy controls

Analysis of the levels of miR-720 shows that it can be used to distinguish normal, healthy controls from all other patient groups (Figure 1A). In particular, miRNA levels are significantly higher in myeloma patients than healthy controls, where the median miRNA concentration in myeloma is 17616 copies per $\mu \mathrm{l}$ compared with 5951 copies per $\mu \mathrm{l}$ in normal subjects $(P<0.001$, Kruskal-Wallis test with Dunn's post test). We also used receiver operating characteristic (ROC) curves, which can be used to determine the true-positive and true-negative rates of a diagnostic test. Figure $2 \mathrm{~A}$ shows that serum miR-720 yielded an AUC (the area under the ROC curve) of $0.9112(P<0.001)$ with $87.2 \%$ sensitivity and $92.3 \%$ specificity for discriminating MGUS and myeloma patients from healthy controls at a cut-off value of 5773 copies per $\mu \mathrm{l}$. Therefore, use of miR-720 alone provides excellent discrimination between healthy subjects and MGUS or myeloma patients.
For miR-1308, the concentration of this miRNA is significantly lower in MGUS and myeloma patients compared with normal healthy controls $(P<0.01$, Figure $1 \mathrm{~B})$. The median concentration of this miRNA in myeloma patients is 157020 copies per $\mu$ l compared with 659640 copies per $\mu \mathrm{l}$ in normal subjects. Similarly to miR720 , it appears that serum miR-1308 is a potential marker for discriminating MGUS and myeloma patients from healthy controls with an AUC of $0.892(P<0.001$; Figure $2 B)$. At a cut-off value of 405400 copies per $\mu \mathrm{l}$, the sensitivity for this marker is $82.1 \%$ and the specificity is $92.3 \%$. The serum concentrations of miR-1246 are not able to distinguish patients with MGUS or myeloma from healthy controls (Figure 2C).

miR-1308 and miR-1246 provide a biomarker signature that can distinguish patients with unrelated illnesses from MGUS and myeloma patients

It is important that the miRNA biomarkers can distinguish patients with MGUS or myeloma from patients with unrelated
A

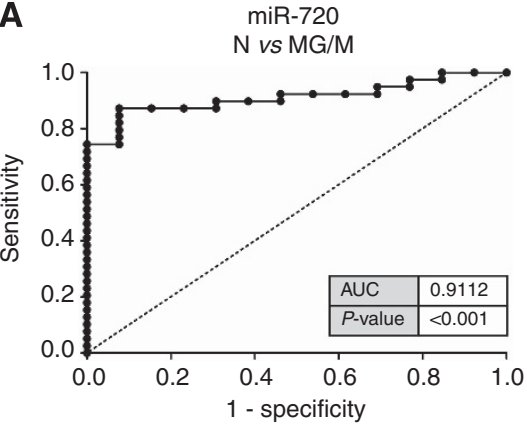

D

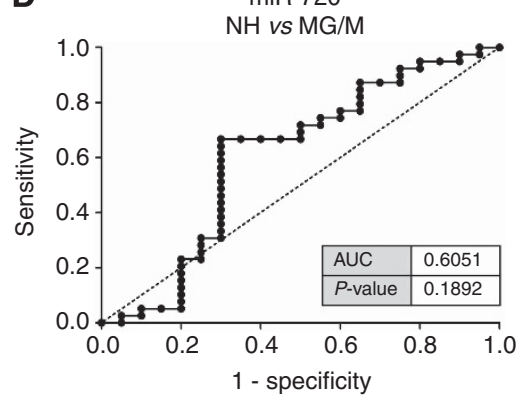

G

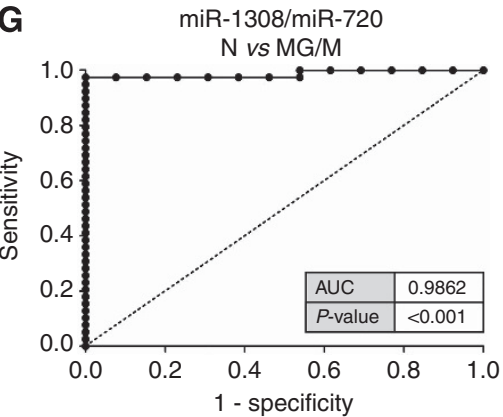

B

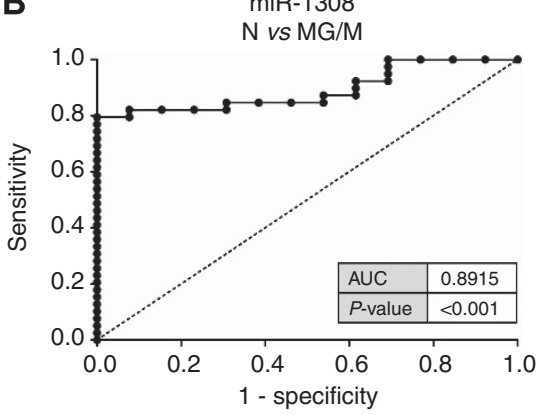

E

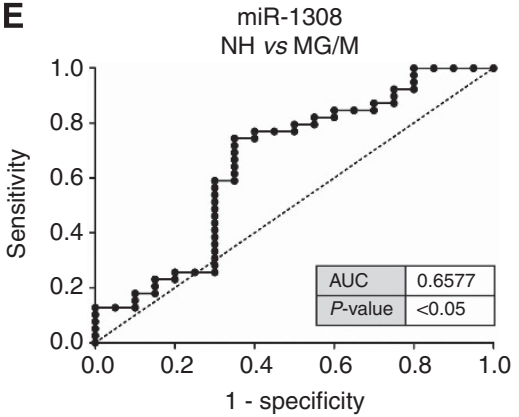

H

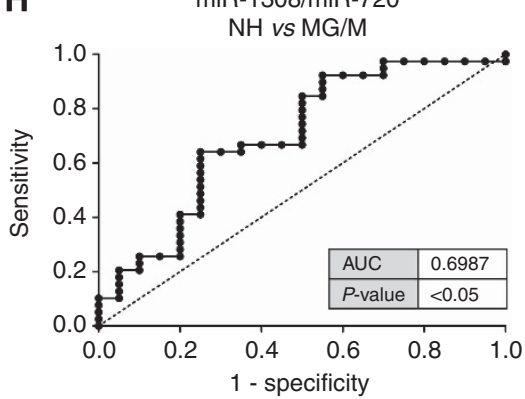

C

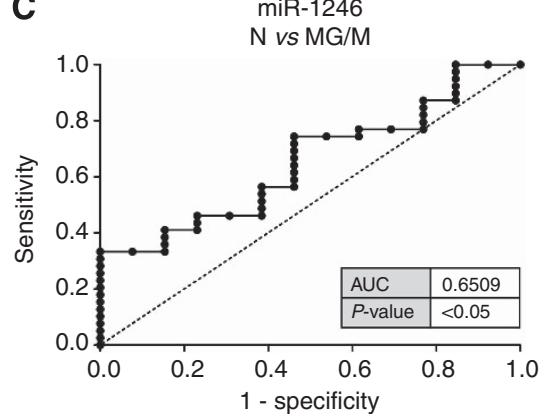

$\mathbf{F}$

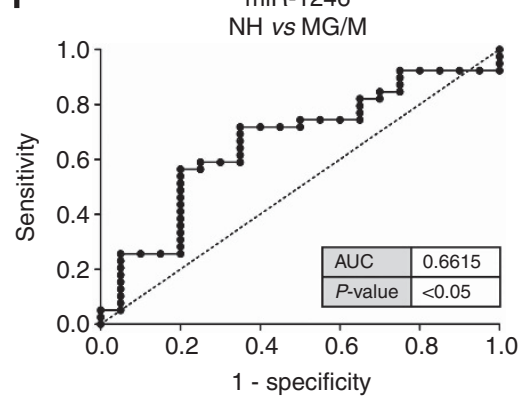

I

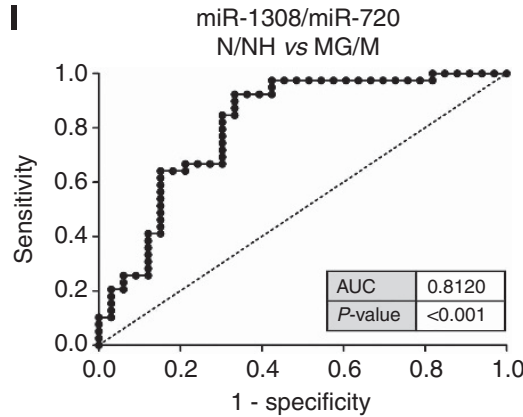

Figure 2 ROC curves to compare the ability of each miRNA, or combinations of miRNAs, to distinguish between different groups. (A) miR-720 can clearly distinguish between $\mathrm{N}$ and MG/M groups $(P<0.00 \mathrm{I})$. (B) miR-I 308 can also distinguish between $\mathrm{N}$ and $\mathrm{MG} / \mathrm{M}$ groups $(P<0.00 \mathrm{I})$. $(\mathbf{C})$ miR- $\mathrm{I} 246$ cannot clearly distinguish between $\mathrm{N}$ and MG/M groups. (D) miR-720 cannot clearly distinguish between NH and MG/M groups. (E) miR-I308 can distinguish between $\mathrm{NH}$ and MG/M groups $(P<0.05)(\mathbf{F})$ miR- 1246 can also distinguish between NH and MG/M groups $(P<0.05)(\mathbf{G})$ The combination of miR-720 and miR-I 308 ([miR-I 308]/[miR-720]) can clearly distinguish between N and MG/M groups $(P<0.05)$. $(\mathbf{H})$ The combination of miR-720 and miR1308 can distinguish between the $\mathrm{NH}$ and MG/M groups $(P<0.05)$. (I) When the $\mathrm{N}$ and $\mathrm{NH}$ groups are considered together, the combination of miR-720 and miR-I 308 can distinguish between the $\mathrm{N} / \mathrm{NH}$ and $\mathrm{MG} / \mathrm{M}$ groups $(P<0.00 \mathrm{I})$. 
illnesses who have some of the symptoms of myeloma, for example, protein in the urine (proteinuria). Such a test would be useful in a hospital setting to aid in the diagnosis of myeloma. Analysis of the levels of serum miR-1308 using a ROC curve shows that it can be used to distinguish MGUS and myeloma from hospitalised patients without these illnesses with an AUC of 0.6577 (sensitivity of $74.4 \%$ and specificity of $65.0 \%$ at a cut-off value of 230850 copies per $\mu \mathrm{l} ; P<0.05$; Figure $2 \mathrm{E}$ ). miR-1246 gives a similar result with a AUC of 0.6615 (sensitivity of $56.4 \%$ and specificity of $80.0 \%$ at a cut-off value of 418150 copies per $\mu \mathrm{l} ; P<0.05$; Figure 2F). Therefore, these biomarkers show potential in distinguishing MGUS and myeloma patients from patients with unrelated illnesses, as well as from healthy controls.

The use of miRNAs in combination provides a more powerful diagnostic tool for distinguishing MGUS and myeloma patients

The use of miR-720 and miR-1308 together provides an even more powerful diagnostic tool for distinguishing normal healthy controls from MGUS and myeloma patients. By dividing the concentration of miR-1308 in the serum by that of miR-720 (i.e., [miR-1308]/[miR-720]), the AUC rises to $0.9862(P<0.001)$ with a sensitivity of $97.4 \%$ and a specificity of $92.3 \%$ at a cut-off value of 83.9 (Figure $2 \mathrm{G}$ ). In other words, this test gives only $7.7 \%$ false positives and $2.6 \%$ false negatives. Therefore, this result shows the great potential of using miRNAs, in combination, to produce predictive diagnostic tests. The miRNAs miR-1308 and miR-720 in combination can also be used to improve the distinction between non-myeloma, non-MGUS patients (NH) and MGUS and myeloma patients $(\mathrm{MG} / \mathrm{M})$. Figure $2 \mathrm{H}$ shows a significant difference between these groups $(P<0.05)$ with the AUC rising to 0.6987 (sensitivity of $64.1 \%$ and a specificity of $75.0 \%$ at a cut-off value of 13.9 ). This combination of miRNAs can also distinguish the normal healthy and non-MGUS, non-myeloma groups (considered together) from the MGUS and myeloma groups. Figure 2I shows that the AUC is $0.8120(P<0.001)$, with a sensitivity of $64.1 \%$ and a specificity of
$84.9 \%$ at a cut-off value of 13.85 . Importantly, these data show that [miR-1308]/[miR-720] could be used as a diagnostic tool for both hospitalised patients as well as for screening apparently healthy individuals.

\section{miR-1246 and miR-1308, in combination, can provide a biomarker signature that has potential to distinguish MGUS from myeloma patients}

One of the key aims of this work is to use miRNAs to distinguish MGUS from myeloma patients. This is the first step on the way to providing a prognostic test that can predict which MGUS patients are likely to progress to myeloma. Our results (Figures 3A-D) show that single miRNAs cannot distinguish the two conditions (Figures $1 \mathrm{~A}-\mathrm{C}$ and Figures $3 \mathrm{~A}-\mathrm{C}$ ). The combination of [miR$1308] /[\mathrm{miR}-720]$ is also unable to make the distinction (Figure 3D). However, the combination of miR-1246 and miR1308 (i.e., concentration of [miR-1246]/[miR-1308]) can distinguish these conditions to some extent (Figure 3E). The ROC plot of [miR-1246]/[miR-1308] shows reasonable separation between the two groups (AUC $=0.7250 ; P<0.05$ ), with a sensitivity of $79.2 \%$ and specificity of $66.7 \%$ at a cut-off value of 6.4$)$. We would expect that analysis of more miRNAs would improve the sensitivity and specificity for distinguishing these patient groups.

\section{The levels of miR-720, miR-1245 and miR-1308 do not change appreciably with age or gender}

One of the attributes of good biomarkers is that they do not change appreciably with age or gender. In order to find out whether these particular miRNAs change with age in the individual patients studied, the copy numbers per $\mu$ l of each of the miRNAs were plotted against age for all patients and controls (Figure 4). When the samples from all groups were considered together, a very slight positive correlation was detected between miR-720 concentration and age (Figure 4A). No significant correlation was observed between miR-1246 or miR-1308 concentration and age for any
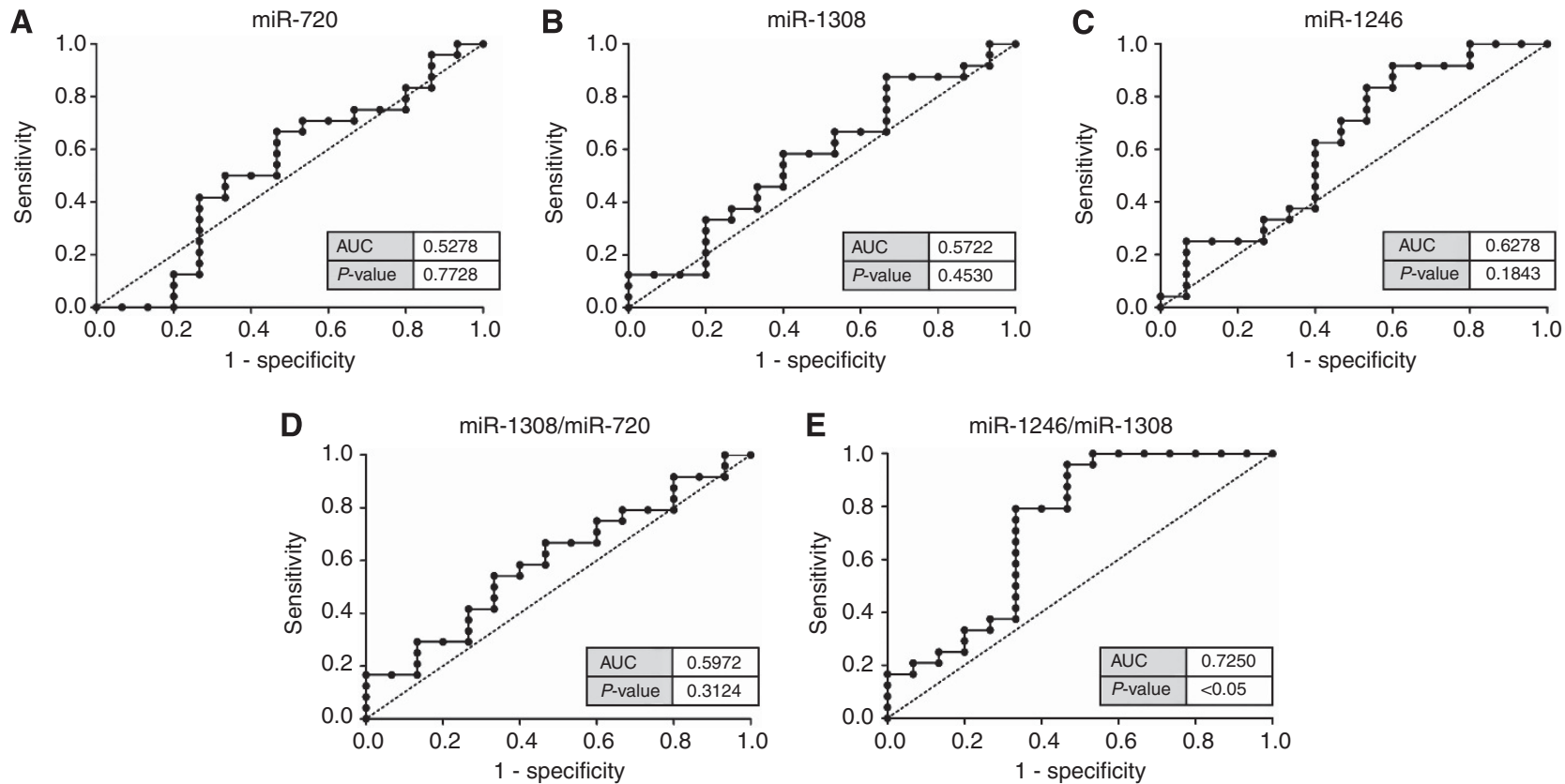

Figure 3 ROC curves to compare the ability of each miRNA, or combinations of miRNAs, to distinguish between the MGUS (MG) and myeloma (M) groups. (A-C) When considered individually, miR-720, miR- I 308 and miR-I246 cannot distinguish between MG and M. (D) The combination of miR- I 308 I miR-720 could distinguish non-MG/M subjects from MG/M subjects (Figure 2), but cannot distinguish between MG and M subjects. (E) The combination of miR-I246/miR-I 308 can distinguish between MG and M, with a sensitivity of $79.2 \%$ and a specificity of $66.7 \%$ at a cut-off value of 6.4 . 

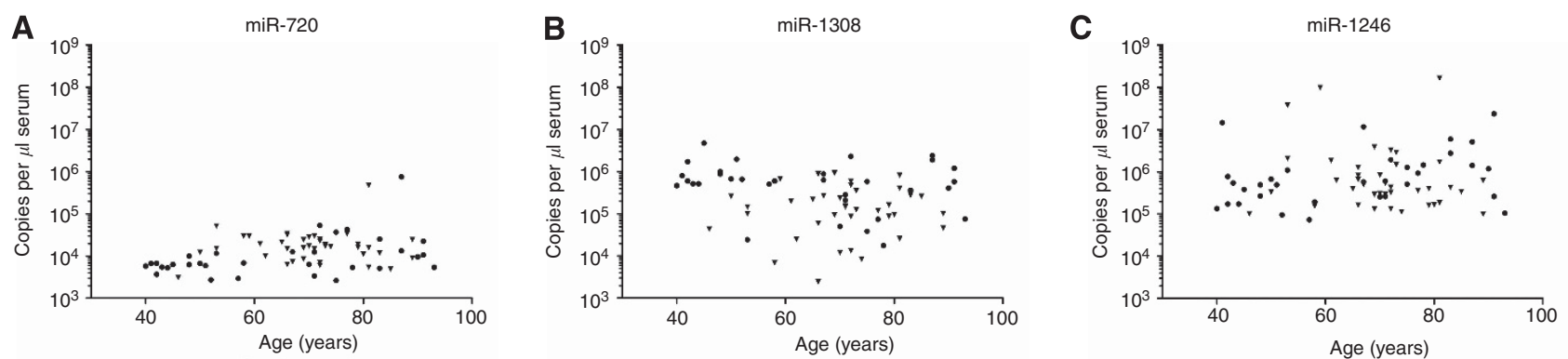

\begin{tabular}{|l|l|l|l|l|}
\cline { 2 - 5 } \multicolumn{1}{c|}{} & Group & Spearman's $r$ & $n$ & $P$-value \\
\cline { 2 - 5 } \multicolumn{1}{c|}{} & All & 0.2702 & 72 & $0.0217^{*}$ \\
\hline$\bullet$ & $\mathrm{N}+\mathrm{NH}$ & 0.3645 & 33 & $0.0370^{*}$ \\
\hline $\boldsymbol{\nabla}$ & $\mathrm{MG}+\mathrm{M}$ & -0.1100 & 39 & 0.5050 \\
\hline
\end{tabular}

\begin{tabular}{|l|l|l|l|l|}
\cline { 2 - 5 } \multicolumn{1}{l|}{} & Group & Spearman's $r$ & $n$ & $P$-value \\
\cline { 2 - 5 } \multicolumn{1}{c|}{ All } & -0.1969 & 72 & 0.0974 \\
\hline • & $\mathrm{N}+\mathrm{NH}$ & -0.2443 & 33 & 0.1706 \\
\hline $\mathbf{\nabla}$ & $\mathrm{MG}+\mathrm{M}$ & 0.0239 & 39 & 0.8850 \\
\hline
\end{tabular}

\begin{tabular}{|l|l|l|l|l|}
\cline { 2 - 5 } \multicolumn{1}{c|}{} & Group & Spearman's $r$ & $n$ & $P$-value \\
\cline { 2 - 5 } \multicolumn{1}{c|}{} & All & 0.1311 & 72 & 0.2722 \\
\hline$\bullet$ & $\mathrm{N}+\mathrm{NH}$ & 0.3582 & 33 & $0.0407^{\text {* }}$ \\
\hline $\mathbf{\nabla}$ & $\mathrm{MG}+\mathrm{M}$ & -0.1495 & 39 & 0.3635 \\
\hline
\end{tabular}
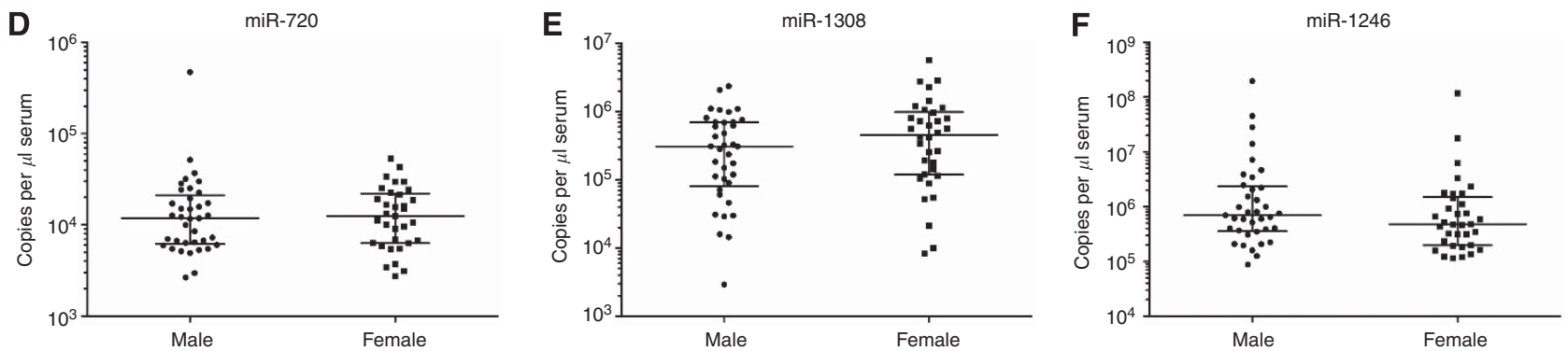

Figure 4 Levels of miR-720 (A), miR-I308 (B) and miR-I246 (C) do not correlate strongly with age. Spearman's rank correlation was used to identify any correlation between miRNA level and age. Any significant correlations were very weak, and significance is lost after correction for multiple significance tests (Bonferroni method). Levels of miR-720 (D), miR-1308 (E) and miR-1246 (F) are not affected by sex. All patients were grouped by sex, and a Mann-Whitney test was used to compare the groups. Graphs show median with interquartile range. $n=37$ for male and $n=34$ for female.

sample groupings even when all samples were considered together (Figures 4A-C). When the samples from all groups were considered together, a very slight positive correlation was detected between miR-720 concentration and age (Figure 4A). No significant correlation was observed between miR-1246 or miR1308 concentration and age for any sample groupings even when all samples were considered together (Figures $4 \mathrm{~B}$ and $\mathrm{C}$ ). The significance of all the weak correlations observed was lost upon correction for multiple significance tests using the Bonferroni method (not shown). Overall, we conclude that any correlation between the expression of these miRNAs and age is too weak to affect the utility of these miRNAs as biomarkers for myeloma.

Similarly, diagnostic tests are simplified if there are no differences in expression of biomarkers between male and female patients. Our data show that the miRNAs tested show no difference in copies per $\mu$ l between men and women for all three miRNAs (Figures $4 \mathrm{D}-\mathrm{F}$ ).

\section{miR-720 and miR-1308 expression levels in serum are not correlated with paraprotein}

The criteria for diagnosis of myeloma includes levels of M-protein in the blood of $>30 \mathrm{gl}^{-1}$ (Swerdlow et al, 2008). However, this is only one aspect of the diagnosis; the severity of the disease and the likelihood of progression to the cancerous state are not particularly well correlated with the amount of paraprotein in the serum. For example, patients with smouldering myeloma do not, by definition, exhibit lytic bone lesions or impaired renal function, which are indicative of myeloma. The clinical data therefore suggest that myeloma is not purely due to mutations resulting in increased paraprotein levels but also requires further mutations to transform the plasma cells to a cancerous state. The amounts of miRNAs we see in circulating blood could reflect that of paraprotein levels or could alternatively provide us with additional information about underlying gene-expression changes that occur upon cancer progression.

Paraprotein and absolute miRNA levels are plotted in Figure 5. These data show that miR-720 and miR-1308 are not correlated with paraprotein in the blood. However, there is slight significant negative correlation of miR-1246 $(P=0.0115)$ with paraprotein. These results suggest that the molecular mechanisms controlling the levels of circulating miR-720 and miR-1308 are not linked to the increased production of paraprotein. However, it is possible that miR-1246 may be involved in, or be repressed by, increased paraprotein production or secretion.

\section{Expression of miR-720 and miR-1246 are correlated}

At present, little is known about the control of secretion of miRNAs into the circulating blood (Reid et al, 2011). However, it is possible that dysregulation of miRNA production or secretion are co-ordinately regulated. To determine whether the concentrations of miR-720, miR-1308 and miR-1246 in serum are co-ordinately regulated, we analysed their correlation with each other. Data presented in Figure 6A show that the expression of miR-720 and miR-1308 is not significantly correlated in any of the patient or control groups. However, levels of miR-720 and miR-1246 do correlate significantly, as an increased concentration of miR-720 is associated with an increase in concentration of miR-1246 in all patient and control groups $(r=0.4648, P<0.001)$. Interestingly, miR-1308 and miR-1246 show a significant positive correlation $(r=0.5524, P<0.001)$ only in MGUS and myeloma patients, whereas no correlation is seen with normal healthy controls and non-MGUS/non-myeloma patients (Figure 6C). It would therefore appear that miR-720 and miR-1246 are co-ordinately regulated, whereas miR-1308 is under different regulatory control. 

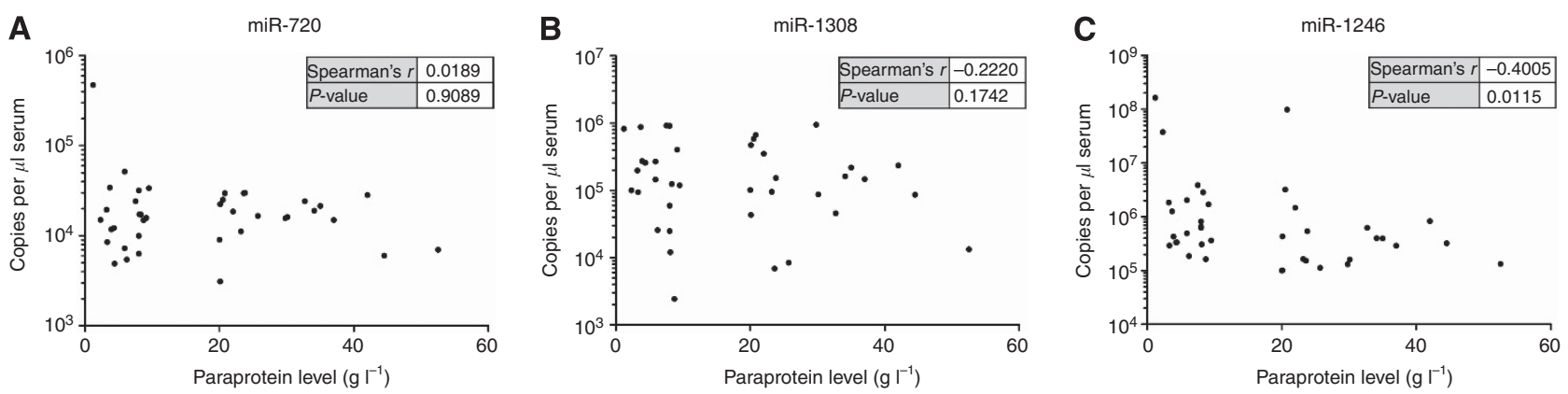

Figure 5 The levels of miR-720 (A) and miR-I308 (B) do not correlate with the level of paraprotein (M-component) in the blood. The level of miR-I 246 (C) shows slight negative correlation with the paraprotein level. Paraprotein levels were only available for the MGUS and myeloma groups and were measured as either $\lg G$ or $\lg A$. Spearman's rank correlation was used to identify correlation between miRNA levels and paraprotein levels.

A

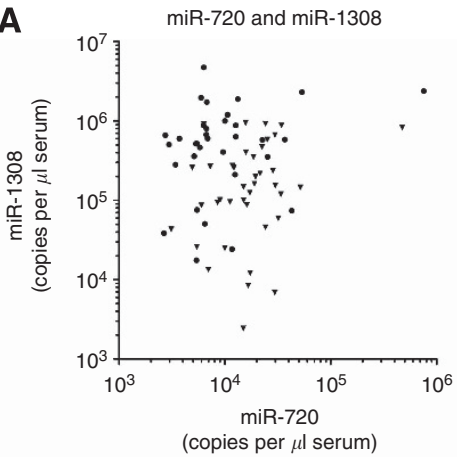

\begin{tabular}{|l|l|l|c|l|}
\cline { 2 - 5 } \multicolumn{1}{c|}{} & Group & Spearman's $r$ & $n$ & $P$-value \\
\cline { 2 - 5 } \multicolumn{1}{c|}{} & All & 0.0170 & 72 & 0.8876 \\
\hline$\bullet$ & $\mathrm{N}+\mathrm{NH}$ & 0.3015 & 33 & 0.0882 \\
\hline $\boldsymbol{\nabla}$ & $\mathrm{MG}+\mathrm{M}$ & 0.2565 & 39 & 0.1150 \\
\hline
\end{tabular}

B

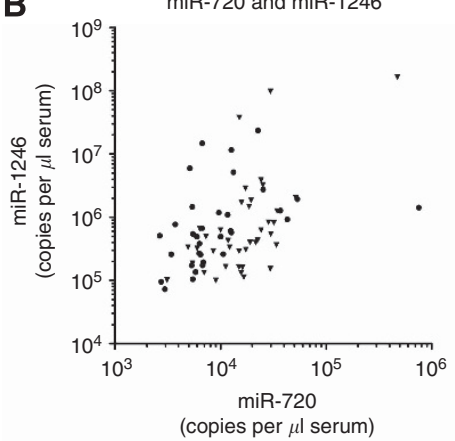

\begin{tabular}{|l|l|l|c|l|}
\cline { 2 - 5 } \multicolumn{1}{c|}{} & Group & Spearman's $r$ & $n$ & $P$-value \\
\cline { 2 - 5 } \multicolumn{1}{c|}{} & All & 0.4648 & 72 & $<0.001^{\star \star *}$ \\
\hline$\bullet$ & $\mathrm{N}+\mathrm{NH}$ & 0.5592 & 33 & $<0.001^{\star \star *}$ \\
\hline $\boldsymbol{\nabla}$ & $\mathrm{MG}+\mathrm{M}$ & 0.5358 & 39 & $<0.001^{\star \star \star}$ \\
\hline
\end{tabular}

C miR-1308 and miR-1246

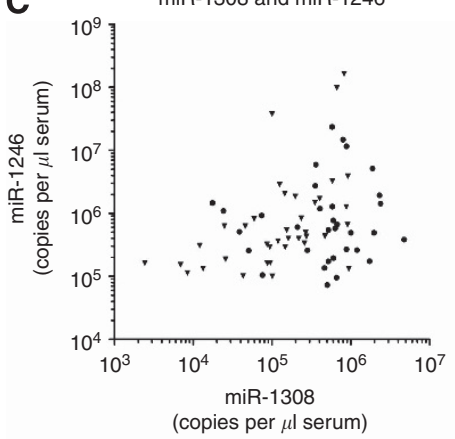

\begin{tabular}{|l|l|c|c|l|}
\cline { 2 - 5 } \multicolumn{1}{c|}{} & Group & Spearman's $r$ & $n$ & $P$-value \\
\cline { 2 - 5 } \multicolumn{1}{c|}{} & All & 0.2988 & 72 & $0.0108^{*}$ \\
\hline$\bullet$ & $\mathrm{N}+\mathrm{NH}$ & 0.0592 & 33 & 0.7437 \\
\hline$\nabla$ & $\mathrm{MG}+\mathrm{M}$ & 0.5524 & 39 & $<0.001^{\star \star *}$ \\
\hline
\end{tabular}

Figure 6 The expression levels of miR-720 and miR-I308 in serum do not show any significant correlation (A), whereas the levels of miR-720 and miR1246 show moderate significant correlation (B). The levels of miR- I 308 and miR- 1246 show moderate significant correlation in MGUS/myeloma (MG + M) subjects, but no significant correlation in non-MGUS/myeloma subjects $(\mathrm{N}+\mathrm{NH})$, and weak correlation overall (C).

\section{DISCUSSION}

In this study, we used microarray analysis, followed by TaqMan qRT-PCR to screen human miRNAs for potential to act as biomarkers in multiple myeloma. The patient groups included patients diagnosed with multiple myeloma, patients with the precancerous condition MGUS, hospitalised patients without MGUS or myeloma and normal, healthy controls. Three miRNAs, miR720, miR-1246 and miR-1308, were identified and then validated in individual patient samples. Use of miR-1308 and miR-720 in combination (i.e., [miR-1308]/[miR-720]) showed excellent specificity and selectivity in distinguishing MGUS/myeloma patients from normal, healthy controls (Figure 2). This combination of miRNAs (i.e., [miR-1308]/[miR-720]) can also be used to distinguish MGUS and myeloma patients from those with unrelated illnesses. Therefore, these miRNAs show great potential for a diagnostic test, which can be used both in the clinic and for screening the general population. We have also shown that miR1246 and miR-1308 in combination (i.e., [miR-1246]/[miR-1308]) can distinguish myeloma from MGUS patients (Figures 2 and 3). This is the first time that serum miRNAs have been shown to act as useful diagnostic and prognostic biomarkers in multiple myeloma.

An unusual aspect of our work is that we performed absolute quantitation of miRNAs in serum to give the number of molecules per $\mu$ l. This allows accurate quantitation of miRNA levels between patient/control samples. Although certain miRNAs, snRNPs or ribosomal RNAs have previously been used for normalisation between serum samples (Reid et al, 2011), we found that these non-coding RNAs were either not detected or changed in levels between patient and control groups. As a suitable normalisation control has not yet been established for use in human serum, the use of absolute quantitation is at present the best way of comparing miRNA levels between individual patients/controls. It is very important that normalisation is performed correctly as use of an inappropriate normaliser may give misleading results and may explain some of the conflicting reports of patterns of miRNA expression that have been given in previous reports.

Our results also show that the distribution of miRNAs detected are similar between MGUS and myeloma patients. This is to be expected as myeloma is a well-known development from the MGUS condition (Kyle and Rajkumar, 2008). However, for all miRNAs, the range of miRNA expression is more widely distributed than for myeloma. This effect could be explained if the onset of myeloma requires increased DNA hypermethylation to shut down more miRNA-encoding genes than in MGUS (similarly to coding genes as previously described (Walker et al, 2012). It is also interesting to note that the miRNAs in serum, which we have found to be useful as a biomarker for myeloma or MGUS, do not overlap with miRNAs within plasma cells that are thought to be associated with the cancerous state (Pichiorri et al, 2008; Lionetti 
et al, 2009; Roccaro et al, 2009). Therefore, it would appear that the miRNAs in serum are either selectively secreted or that the miRNAs in serum are produced by other cells within the body.

Our data also show that miR-720 and miR-1308 levels are not significantly correlated with the levels of paraprotein in the blood (Figure 5). These data suggest that miR-720 and miR-1308 are providing different information on the pre-cancerous and cancerous state that is not related to the dysregulation of gene expression, resulting in overexpression of paraprotein. Because high levels of $\mathrm{M}$-component in the blood are not always related to the cancerous state, as is the case in smouldering myeloma, these miRNA biomarkers are likely to be providing alternative and potentially useful information on the progression of the disease.

The miRNAs that we have found to be dysregulated in MGUS and myeloma patients have not yet been found in the circulating blood in association with other cancers. This is an advantage as far as a diagnostic test is concerned because these miRNAs are more likely to provide an extremely specific test for MGUS and myeloma. In contrast, some miRNAs, such as miR-155, have been shown to be upregulated in the sera of patients with a number of different cancers, including diffuse large B-cell lymphoma and breast cancer (Faraoni et al, 2009; Roth et al, 2010). The mRNA targets of the miRNAs that we have detected have not yet been experimentally determined, but miR-720 and miR-1246 have an association with control of apoptosis, suggesting that they could be shed by cells undergoing programmed cell death (Chikh et al, 2011).

Analysis of our data has shown that miR-1308 is expressed in a different pattern than miR-720 and miR-1246 in that the levels of this miRNA generally decrease (rather than increase) in MGUS and myeloma patient sera compared with controls (Figure 1). In addition, the expression of miR-1308 is not correlated with that of miR-720 or miR-1246 (Figure 6). It is now known that miR-1308 is not a miRNA but is a $5^{\prime}$-cleaved fragment of a GlyGCC tRNA. The presence of modified ribonucleosides in the serum of humans and

\section{REFERENCES}

Borek E, Baliga BS, Gehrke CW, Kuo CW, Belman S, Troll W, Waalkes TP (1977) High turnover rate of transfer RNA in tumor tissue. Cancer Res 37: $3362-3366$

Chen X, Liang H, Zhang J, Zen K, Zhang CY (2012) Secreted microRNAs: a new form of intercellular communication. Trends Cell Biol 22: $125-132$

Chikh A, Matin RN, Senatore V, Hufbauer M, Lavery D, Raimondi C, Ostano P, Mello-Grand M, Ghimenti C, Bahta A, Khalaf S, Akgul B, Braun KM, Chiorino G, Philpott MP, Harwood CA, Bergamaschi D (2011) iASPP/p63 autoregulatory feedback loop is required for the homeostasis of stratified epithelia. EMBO J 30: 4261-4273

Faraoni I, Antonetti FR, Cardone J, Bonmassar E (2009) miR-155 gene: a typical multifunctional microRNA. Biochim Biophys Acta 1792: 497-505

Git A, Dvinge H, Salmon-Divon M, Osborne M, Kutter C, Hadfield J, Bertone P, Caldas C (2010) Systematic comparison of microarray profiling, real-time PCR, and next-generation sequencing technologies for measuring differential microRNA expression. RNA 16: 991-1006

Heneghan HM, Miller N, Kelly R, Newell J, Kerin MJ (2010) Systemic miRNA-195 differentiates breast cancer from other malignancies and is a potential biomarker for detecting noninvasive and early stage disease. Oncologist 15: 673-682

Hunter MP, Ismail N, Zhang X, Aguda BD, Lee EJ, Yu L, Xiao T, Schafer J, Lee ML, Schmittgen TD, Nana-Sinkam SP, Jarjoura D, Marsh CB (2008) Detection of microRNA expression in human peripheral blood microvesicles. PLoS One 3: e3694

Jones CI, Newbury SF (2010) Functions of microRNAs in Drosophila development. Biochem Soc Trans 38: 1137-1143

Kong YW, Ferland-McCollough D, Jackson TJ, Bushell M (2012) microRNAs in cancer management. Lancet Oncol 13: e249-e258

Kyle RA, Rajkumar SV (2008) Multiple myeloma. Blood 111: 2962-2972

Kyle RA, Remstein ED, Therneau TM, Dispenzieri A, Kurtin PJ, Hodnefield JM, Larson DR, Plevak MF, Jelinek DF, Fonseca R, Melton 3rd LJ, mice (Borek et al, 1977; Speer et al, 1979) has previously suggested that tRNA fragments may be present in the circulating blood. However, our data show, for the first time, that the intact $5^{\prime}$ half of a tRNA is present in human serum. Our finding that this cleaved tRNA is differentially expressed in the serum of myeloma and MGUS patients compared with controls is entirely novel and shows that these cleaved tRNAs also have potential as diagnostic and prognostic biomarkers.

The miRNA signature that we have identified for myeloma also has other potential benefits for myeloma patients. For example, this serum miRNA signature could be useful to pharmaceutical companies for monitoring patient responses to new drug treatments or regimes. In addition, the miRNA signature could be used for myeloma stratification, prognosis estimation, prediction of therapeutic efficacy, maintenance of surveillance following treatment or forecasting of disease recurrence. Finally, this study sets the stage for future analysis of other potential miRNA biomarkers in a variety of ethnic groups in order to improve prognosis/diagnosis of myeloma and predictions of therapeutic responses.

\section{ACKNOWLEDGEMENTS}

We thank Clare Rizzo-Singh, Karen Scruby (both funded by the BBSRC) and Betty Gration (a summer student funded by the Biochemical Society) for their technical help. We also wish to thank Tom Rider, an Academic Clinical Fellow funded by NIHR, for his help with the patient data and Stephanie Goubet, a statistician funded by Brighton and Sussex Medical School, for her statistical advice. This work was funded by the University of Sussex Enterprise Fund, the Biotechnology and Biological Sciences Research Council (BB/I021345/1 and BB/I007989/1), the Elimination of Leukaemia Fund and the Biochemical Society (Summer Vacation Studentship).
Rajkumar SV (2007) Clinical course and prognosis of smoldering (asymptomatic) multiple myeloma. $N$ Engl J Med 356: 2582-2590

Lionetti M, Biasiolo M, Agnelli L, Todoerti K, Mosca L, Fabris S, Sales G, Deliliers GL, Bicciato S, Lombardi L, Bortoluzzi S, Neri A (2009) Identification of microRNA expression patterns and definition of a microRNA/mRNA regulatory network in distinct molecular groups of multiple myeloma. Blood 114: e20-e26

Lodes MJ, Caraballo M, Suciu D, Munro S, Kumar A, Anderson B (2009) Detection of cancer with serum miRNAs on an oligonucleotide microarray. PLoS One 4: e6229

McKenna RW, Kyle RA, Kuehl WM, Grogan TM, Harris NL, Coupland RW (2008) Plasma cell neoplams. In WHO classification of tumours of haematopoietic and lymphoid tissues, Swerdlow SH, Campo E, Harris NL, Jaffe ES, Pileri SA, Stein H, Thiele J, Vardiman JW (eds) pp 200-203. International Agency for Research on Cancer: Lyon

Mitchell PS, Parkin RK, Kroh EM, Fritz BR, Wyman SK, PogosovaAgadjanyan EL, Peterson A, Noteboom J, O'Briant KC, Allen A, Lin DW, Urban N, Drescher CW, Knudsen BS, Stirewalt DL, Gentleman R, Vessella RL, Nelson PS, Martin DB, Tewari M (2008) Circulating microRNAs as stable blood-based markers for cancer detection. Proc Natl Acad Sci USA 105: 10513-10518

Neal CS, Michael MZ, Pimlott LK, Yong TY, Li JY, Gleadle JM (2011) Circulating microRNA expression is reduced in chronic kidney disease. Nephrol Dial Transplant 26: 3794-3802

Pichiorri F, Suh SS, Ladetto M, Kuehl M, Palumbo T, Drandi D, Taccioli C, Zanesi N, Alder H, Hagan JP, Munker R, Volinia S, Boccadoro M, Garzon R, Palumbo A, Aqeilan RI, Croce CM (2008) MicroRNAs regulate critical genes associated with multiple myeloma pathogenesis. Proc Natl Acad Sci USA 105: $12885-12890$

Reid G, Kirschner MB, van Zandwijk N (2011) Circulating microRNAs: association with disease and potential use as biomarkers. Crit Rev Oncol Hematol 80: 193-208 
Roccaro AM, Sacco A, Thompson B, Leleu X, Azab AK, Azab F, Runnels J, Jia X, Ngo HT, Melhem MR, Lin CP, Ribatti D, Rollins BJ, Witzig TE, Anderson KC, Ghobrial IM (2009) MicroRNAs 15a and 16 regulate tumor proliferation in multiple myeloma. Blood 113: 6669-6680

Roth C, Rack B, Muller V, Janni W, Pantel K, Schwarzenbach H (2010) Circulating microRNAs as blood-based markers for patients with primary and metastatic breast cancer. Breast Cancer Res 12: R90

Speer J, Gehrke CW, Kuo KC, Waalkes TP, Borek E (1979) tRNA breakdown products as markers for cancer. Cancer 44: 2120-2123

Swerdlow SH, Campo E, Harris NL, Jaffe ES, Pileri SA, Stein H, Thiele J, Vardiman JW (eds) (2008) WHO classification of tumours of Haematopoietic and lymphoid tissues. I.A.R.C.: Lyon

Terpos E, Rahemtulla A (2005) Myeloma. In Postgraduate Haematology, Hoffbrand AV, Catovsky D, Tuddenham EGD (eds) 5th edn pp 681-702. Blackwell: Oxford
Walker BA, Wardell CP, Melchor L, Hulkki S, Potter NE, Johnson DC Fenwick K, Kozarewa I, Gonzalez D, Lord CJ, Ashworth A, Davies FE, Morgan GJ (2012) Intraclonal heterogeneity and distinct molecular mechanisms characterize the development of $t(4 ; 14)$ and $t(11 ; 14)$ myeloma. Blood 120: 1077-1086

Zen K, Zhang C-Y (2010) Circulating MicroRNAs: a novel class of biomarkers to diagnose and monitor human cancers. MED RES REV 32: 326-348

Zhu W, Qin W, Atasoy U, Sauter E (2009) Circulating microRNAs in breast cancer and healthy subjects. BMC Res Notes 2: 89

cC)(ㄱ) This work is licensed under the Creative Commons (c) $\mathrm{BY}$ NC SA Attribution-NonCommercial-Share Alike 3.0 Unported License. To view a copy of this license, visit http://creativecommons.org/ licenses/by-nc-sa/3.0/ 